\section{Management of acute osteomyelitis: a ten-year experience}

\author{
Caitlin Helm, Emily Huschart, Rajat Kaul, \\ Samina Bhumbra, R. Alexander Blackwood, \\ Deepa Mukundan \\ Divisions of Pediatric Infectious Diseases, \\ University of Michigan and University of \\ Toledo; Office for Health Equity and \\ Inclusion, University of Michigan Medical \\ School, USA
}

\section{Abstract}

Osteomyelitis is an infection of the bone; proper management requires prolonged antibiotic treatment. Controversy exists as to when a patient should transition from intravenous to oral antibiotics. However, due to the high bioavailability of some oral antibiotics, optimal time to transition from high to low bioavailability antibiotics is a more valid consideration. Additionally, there are questions surrounding the efficacy of certain antibiotics, specifically trimethoprim-sulfamethoxazole (TMP-SMX), in treating osteomyelitis. After obtaining Institutional Review Board approval from both universities, a retrospective chart review was conducted, utilizing an author-created severity scale, on all patients seen by Pediatric Infectious Diseases at the Universities of Michigan and Toledo with an acute osteomyelitis diagnosis from 2002-2012. There were 133 patients, 106 treated successfully. Success was defined in this study specifically as treatment of $<14$ weeks without recurrence within 30 days of stopping antibiotics or permanent site disability. Seventeen patients were treated with TMP-SMX at comparable cure rates. Patients with pre-existing bone defects (noted in radiological reports), initial erythrocyte sedimentation rate $(\mathrm{ESR}) \geq 70$, hematogenous osteomyelitis with soft tissue extension, and skull osteomyelitis were associated with increased failure rate. Switch to low bioavailability antibiotics occurred, on average, at 3.5 weeks; however, switching before then was not associated with decreased cure rate. As prevalence of methicillin-resistant Staphylococcus aureus (MRSA), especially clindamycin-resistant MRSA, increases, TMP-SMX appears to be an acceptable antibiotic. There does not appear to be a minimum length of high bioavailability treatment required for cure. Prior bone defect, extensive infection, $\mathrm{ESR} \geq 70$, or skull osteomyelitis may be indications for more aggressive management.

\section{Introduction}

Osteomyelitis is an infection of the bone and/or bone marrow. In children it is typically found in one of the long bones and management requires prolonged antibiotic courses with or without surgical debridement. ${ }^{1-6}$ While there is some literature suggesting that 3 weeks of antibiotic treatment is sufficient for most acute osteomyelitis cases, pediatric patients at the Universities of Michigan and Toledo are typically treated for 4-6 weeks. ${ }^{2}$ The pathogenesis of osteomyelitis is either via hematogenous seeding, which is more common in pediatric patients, or an extension from a contiguous infection. ${ }^{1-3,5}$ The most common pathogen in osteomyelitis is Staphylococcus aureus, although a variety of organisms can cause this disease. ${ }^{1-5,7,8}$ There are 3 classifications of osteomyelitis: acute duration of infection prior to treatment is less than 2 weeks, subacute - duration of infection prior to treatment is 2 weeks to approximately 3 months, or chronic - duration of infection prior to treatment is greater than 3 months. ${ }^{2,4,5}$ An acute or subacute infection can progress to chronic osteomyelitis due to treatment failure as well. For the purposes of this study, we are investigating acute infections. While osteomyelitis is not a new or exceedingly rare condition, there is no consensus as to the best medical management of this disease. ${ }^{4,9}$ Controversy exists with regards to intravenous (IV) versus oral ( $\mathrm{PO})$ medication, more specifically as to the ideal length of each treatment and when (or if) the transition from IV to PO medication should occur., ${ }^{4,8,10,11}$ Transitioning from IV to PO has many advantages including: reduction in economic burden for the patient because $\mathrm{PO}$ antibiotics are less expensive than IV as well as shorter inpatient hospital stays because a medical professional is not required to administer antibiotics. ${ }^{12}$ Additionally, prolonged use of a central venous catheter or a PICC line is noted in the literature to be associated with increased risk of additional infections. ${ }^{13}$ However, due to the high bioavailability, defined as Fraction of a dose of drug that is absorbed from its site of administration and reaches, in an unchanged form, the systemic circulation, of some of the oral antibiotics used to treat osteomyelitis, the optimal time to transition from high (IV \pm high bioavailable P0) to low bioavailability antibiotics is a more valid consideration. ${ }^{12,14,15}$ This concept has not been extensively studied in the literature, instead focusing on the transition from IV to PO; therefore, this study aims to begin the conversation about the role of bioavailability in pediatric acute osteomyelitis treatment.

With the emergence of clindamycin resistant methicillin-resistant $S$. aureus (MRSA), the efficacy of trimethoprim-sulfamethoxazole
Correspondence: Deepa Mukundan, Divisions of Pediatric Infectious Diseases, University of Toledo, 2222 Cherry Street, \#2300, Toledo, OH 43608, USA.

Tel: +1.419.251.8039.

E-mail: Deepa.Mukundan@utoledo.edu

Key words: Acute osteomyelitis; trimethoprimsulfamethoxazole; bioavailability; debridement; ESR.

Conflict of interest: the authors declare no potential conflict of interest.

Received for publication: 4 December 2015.

Revision received: 6 May 2016.

Accepted for publication: 1 June 2016.

This work is licensed under a Creative Commons Attribution-NonCommercial 4.0 International License (CC BY-NC 4.0).

(C) Copyright C. Helm et al., 2016

Licensee PAGEPress, Italy

Infectious Disease Reports 2016; 8:6350

doi:10.4081/idr.2016.6350

(TMP-SMX), which has high bioavailability as a PO antibiotic, has begun to be considered to be a viable treatment option for pediatric acute osteomyelitis and needs to be studied further. ${ }^{14,15}$

The objectives of this study were to identify in pediatric patients with acute osteomyelitis: criteria that indicate an increased likelihood of treatment failure, the optimal time to transition from high to low bioavailability, and the efficacy of TMP-SMX for the treatment of acute osteomyelitis in pediatric patients.

\section{Materials and Methods}

After Institutional Review Board (IRB) approval was obtained at both institutions, a retrospective chart review was conducted on all patients seen by the Pediatric Infectious Disease Divisions at the Universities of Michigan and Toledo with a diagnosis of acute osteomyelitis from 2002 to 2012.

Diagnosis of acute osteomyelitis during this time period was the only criteria for entry in the study; age was not explicitly a rule out criteria, but no patient above the age of 21 was included because both divisions examined were pediatric divisions. The acute osteomyelitis patients were identified via query of electronic medical records via ICD-9 codes. Patients were not explicitly diagnosed in this study, but rather they were identified by the ICD-9 code search and were included unless there was clear evidence of an incorrect classification upon chart review; for example, if explicitly stated that there was a period of $>2$ 
weeks between infection and treatment or if there was no evidence of any bone infection. Patient demographics (age, gender, race, etc.), co-morbidities, pathogenesis, laboratory values, pathogens (determined via synovial fluid, bone, or blood culture), imaging, surgeries, antibiotic treatment course, recurrence of infection, complications, and success of treatment were analyzed. Statistics were performed using chi square analyses and independent ttests in collaboration with a biostatistician from the University of Toledo.

Ultimately, all acute osteomyelitis patients were categorized retrospectively via a new severity scale created specifically for this study based on three criteria - pathogenesis, extent of infection (determined via imaging), and whether surgical debridement was performed. These criterion were chosen because they are part of the clinical decision making process in determining treatment type and duration. Pathogenesis specifically was divided into two categories - hematogenous or contiguous. The case then received a number that described the extent of the infection. For hematogenous osteomyelitis there were five possible stages in the new severity scale: stage 1 - the infection was confined to the bone marrow; stage 2 - the infection extended past the bone marrow, but was confined to the bone; stage 3 - there was evidence of osteosclerosis, but the infection was still confined to the bone; stage 4 there was soft tissue involvement in addition to the bone infection; stage 5 - the soft tissue involvement was extensive. Contiguous cases were divided into 2 possible stages: stage 1 the infection was confined to the bone marrow or stage 2 - there was evidence of osteosclerosis. Stage was defined by radiological imaging, most frequently this was imaging was an MRI, but patients who did not have an MRI performed were classified via X-Ray or computed tomography imaging. All cases were further designated $a$ - no surgical debridement or $b$ $\geq 1$ surgical debridement.

Patients were considered to have completed treatment successfully, for the purposes of this study, if they completed a treatment course of $\leq 14$ weeks without permanent disability to the affected site. Fourteen weeks was chosen as the cutoff point to accommodate for patients who may have had a borderline subacute infection or prior bone infections, standard treatment for which can last up to approximately 12 weeks, with a leeway of 2 weeks to account for any elongated treatments due to scheduling difficulties. Permanent disability was defined as continued abnormalities in function, feeling, or significant deformity due to infection as noted in the patient's chart in follow-up appointments.

\section{Results}

\section{Patient demographics}

A total of 133 patients were enrolled in the study, 87 from the University of Michigan and 46 from the University of Toledo, 106 (79.7\%) of whom were treated were defined as successes by this study's criteria with comparable rates seen between Michigan (75.9\%) and Toledo (87\%). Overall, there were more males than females (53.4 to $46.6 \%$ ); approximately $75 \%$ of the patients were Caucasian, $12 \%$ African American, less than 1\% Asian, and nearly $13 \%$ other/not reported. The mean age of the patients was just under 9 years $(8.93 \pm 0.45)$, overall ranging from 20 days - 21 years old (Table 1).

The most common sites of osteomyelitis were: $24.8 \%$ lower leg (tibia, fibula, and ankle), 21.1\% upper leg (femur and patella), and $18 \%$ pelvis (Table 2). Skull osteomyelitis was also associated with increased failure rate (Table 2; $\mathrm{P}=0.0012$ ).

S. aureus accounted for $58.6 \%(\mathrm{n}=78)$ of all infections with MRSA specifically accounting for $32.1 \%$ of the $S$. aureus infections and approximately $18.8 \%$ of all of the infections $(\mathrm{n}=25$; Table 3$)$. There was no significant difference in the cure rate between MRSA and

Table 1. Patient demographics.

\begin{tabular}{|c|c|c|c|}
\hline & $\begin{array}{c}\text { Michigan, } \\
n=87\end{array}$ & $\begin{array}{c}\text { Toledo, } \\
\mathrm{n}=46\end{array}$ & $\begin{array}{c}\text { Overall, } \\
n=133\end{array}$ \\
\hline Cured & $75.9 \%$ & $87 \%$ & $79.7 \%$ \\
\hline Hematogenous & $88.5 \%$ & $65.2 \%$ & $53.4 \%$ \\
\hline Male & $47.1 \%$ & $65.2 \%$ & $53.4 \%$ \\
\hline Average age & 8.82 yrs. & $9.15 \mathrm{yrs}$. & 8.91 yrs. \\
\hline Caucasian & $83.9 \%$ & $58.7 \%$ & $74.6 \%$ \\
\hline African American & $9.2 \%$ & $15.2 \%$ & $11.9 \%$ \\
\hline Asian & $1.1 \%$ & $0.0 \%$ & $0.8 \%$ \\
\hline Other/not reported & $5.7 \%$ & $26.1 \%$ & $12.7 \%$ \\
\hline
\end{tabular}

Table 2. Location of osteomyelitis and cure.

\begin{tabular}{lcc} 
Location & Osteomyelitis, total=133 n (\%) & Cure rate, $\mathrm{n} /$ total (\%) \\
Skull & $6(4.5)$ & $2 / 6(33.3)^{*}$ \\
Spine & $4(3.0)$ & $3 / 4(75)$ \\
\hline Thorax & $2(1.5)$ & $1 / 2(50)$ \\
Pelvis & $24(18.0)$ & $20 / 24(83.3)$ \\
\hline Arm & $7(5.3)$ & $6 / 7(85.7)$ \\
Hand & $8(6.0)$ & $7 / 8(87.5)$ \\
\hline Upper leg & $28(21.1)$ & $22 / 28(78.6)$ \\
Lower leg & $33(24.8)$ & $28 / 33(84.8)$ \\
\hline Foot & $18(13.5)$ & $14 / 18(77.8)$ \\
Multifocal & $3(2.3)$ & $3 / 3(100)$ \\
\hline All & $133(100)$ & $106 / 133(79.7)$ \\
\hline
\end{tabular}

*P=0.0012; comparing particular site cure rate versus overall cure rate.

Table 3. Length of treatment and cure.

\begin{tabular}{lccc} 
& N. patients & N. cured (\%) & Average length of treatment in weeks \\
I\&D & 80 & $60(75)$ & $11.4^{*}$ \\
No I\&D & 53 & $46(86.8)$ & 8 \\
\hline$E S R<50$ & 72 & $62(86.1)$ & 8.9 \\
$E S R<70$ & 97 & $85(87.6)$ & 9.3 \\
\hline$E S R \geq 70$ & 31 & $21(67.7)$ & 12.2 \\
Hematogenous & 107 & $88(82.2)$ & 9.23 \\
\hline Contiguous & 26 & $18(69.0)$ & 13.21 \\
\hline *P $=0.015$. I\&D, Irrigation and Debridement: ESR, erythrocyte sedimentation rate. Note: 5 patients never had ESR rates measured
\end{tabular}

*P=0.015. I\&D, Irrigation and Debridement; ESR, erythrocyte sedimentation rate. Note: 5 patients never had ESR rates measured 
methicillin sensitive $S$. aureus infections ( $\mathrm{P}=0.297$; Table 4). Other microorganisms included: non-specific gram-positive organisms $(\mathrm{n}=9)$, non-specific gram-negative organisms $(n=18)$, streptococcus $(n=12)$, anaerobic $(n=3)$, pro acne $(n=1)$, and coagulase negative staphylococci $(n=12)$. Multiple patients had cultures that were positive for $2+$ microorganisms $(\mathrm{n}=17)$ and multiple had negative cultures $(n=24)$.

Of the patients, $44.4 \%$ who failed treatment were noted to have a prior bone defect on radiology reports when compared with patients who were successfully treated (7.5\%; $\mathrm{P}<0.0001)$.

\section{Pathogenesis and cure}

There were 26 contiguous patients and 107 hematogenous patients. Hematogenous infections confined to the bone (stages 1-3) approached statistical significance in association with a higher cure rate than infections with soft tissue involved (stages 4-5; $\mathrm{P}<0.09$; Table 5). The association between extension into surrounding soft tissue and treatment failure was even more pronounced when surgery was required in patients with hematogenous osteomyelitis $(\mathrm{P}<0.02$; Table 5$)$.

\section{Length of treatment and cure}

There was no statistically significant difference in cure rate or length of treatment based on the pathogenesis (hematogenous vs. contiguous) with an average of $13.21 \pm 2.64$ weeks of treatment for contiguous infections versus an average of $9.23 \pm 0.68$ weeks for hematogenous $(\mathrm{P}=0.128$ and 0.458 respectively compared to the mean treatment length of 10.01 weeks; Table 3) Patients who had at least one irrigation and debridement (I\&D) had a statistically significant increase in treatment length $(\mathrm{P}=0.015$; Table 3$)$, on average received nearly 3.5 weeks longer of antibiotic therapy and had a decreased cure rate which approached significance ( $\mathrm{P}=0.071$; Table 5); however, additional I\&Ds did not further increase failure rate.

While only $24.2 \%$ ( $n=32)$ of the patients had an initial $E S R \geq 70$ (nearly 3.5 times the rate of the normal value of $<20$, signifying a huge immunologic response), $40 \%$ of the treatment failures had $E S R \geq 70$ compared to $12.6 \%$ of the treatment successes $(p=0.073)$. Additionally, an initial $\mathrm{ESR} \geq 70$ corresponded with a borderline increase in treatment failure as well as almost 3 weeks longer treatment that patients with an $E S R<70$. Patients with both an $E S R \geq 70$ and an infection with soft tissue involvement ( $\geq$ hematogenous stage 4 ) have an increased failure rate compared to patients with an ESR $<70$ and an infection without soft tissue involvement $(\mathrm{P}<0.02)$. For unknown reasons, seven patients were treated with antibiotics for less than the standard 6 weeks, of these, 6 had an initial ESR below 50. An ESR $<50$ corresponds with, on average, approximately 3.5 weeks less treatment than an ESR $\geq 70$ (Table 3). 84 patients were treated with only high bioavailability antibiotics (defined as all IV antibiotics or oral: clindamycin, bactrim, linezolid, quinolone, fluconazole, and doxycycline; we acknowledge that not all of these antibiotics are traditional first-line osteomyelitis treatment choices, some of these antibiotics were chosen due to co-morbidities, antibiotic insensitivities found on culture, or physician preference) with a cure rate of $80 \%$. Thirteen patients were transitioned from high bioavailability to low bioavailability (oral: cephalosporin or penicillin) antibiotics in 2 weeks or less, with a cure rate of $92.3 \%(\mathrm{P}=0.484)$. Transition after 3-6 weeks of high bioavailability treatment had a comparable cure rate (90\%) in both groups.
Late transition, following more than 6 weeks of high bioavailability treatment, had a cure rate of $25 \%(\mathrm{P}<0.01)$. There were several patients who received combination treatments, defined as patients who received high and low bioavailability antibiotics at the same time. Nine patients received less than 3 weeks of high bioavailability antibiotics, then combination treatment for the rest of their antibiotic course with a comparable cure rate (77.8\%) to transitioning to low bioavailability treatment after 3-6 weeks. Three patients received greater than or equal to 4 weeks of high bioavailability antibiotic treatment before transitioning to combination treatment with only a $33 \%$ cure rate; however, there was no statistically significant difference $(\mathrm{P}=0.557)$ between this transition and transitioning to low bioavailability antibiotics at $\geq 4$ weeks.

\section{Therapy with trimethoprim-sul- famethoxazole}

Eighteen patients in total were treated with $\geq 7$ days of TMP-SMX. However, one of these patients did not begin TMP-SMX treatment until after failing a course of 6 weeks of clindamycin + rifampin. Although this patient later responded to TMP-SMX therapy, the patient was determined to have progressed to chronic osteomyelitis due to the failure of the first treatment course and, therefore, will be excluded from subsequent analysis of TMPSMX efficacy. Fifteen of the now 17 patients treated with TMP-SMX (88.2\%) were cured, which is comparable to the overall study cure rate $(79.7 \%)$. Of the two patients who failed

Table 4. Pathogens and cure.

\begin{tabular}{lcc} 
Pathogen & Occurrence rate, $\mathbf{n}(\%)$ & Cure, n (\%) \\
MRSA & $25(18.8)$ & $19(76)$ \\
MSSA & $53(39.9)$ & $45(84.9)$ \\
\hline Strep (all strains grouped) & $12(9.0)$ & $9(75.0)$ \\
Gram negative & $18(13.5)$ & $14(77.8)$ \\
\hline
\end{tabular}

MRSA, Methicillin-resistant Staphylococcus aureus; MSSA, methicillin sensitive Staphylococcus aureus.

Table 5. Hematogenous stage and cure.

\begin{tabular}{lccc} 
Stage & Overall cured (\%) & $\begin{array}{c}\text { Cured " } a \text { " } \\
\text { Patients } \\
\text { without debridement (\%) }\end{array}$ & $\begin{array}{c}\text { Cured " } \mathrm{b} \text { " } \\
\text { Patients with> } 1 \text { debridement }(\%)\end{array}$ \\
1 & $6 / 9(66.7)$ & $1 / 4(25)$ & $5 / 5(100)$ \\
2 & $34 / 36(94.7)$ & $17 / 18(94.4)$ & $17 / 18(94.4)$ \\
\hline 3 & $4 / 4(100)$ & $1 / 1(100)$ & $3 / 3(100)$ \\
4 & $37 / 46(80.4)$ & $15 / 16(93.8)$ & $22 / 30(73.3)$ \\
\hline 5 & $7 / 12(58.3)$ & $2 / 3(66.7)$ & $5 / 9(55.6)$ \\
Total & $88 / 107(82.6)$ & $36 / 42(85.7)$ & $52 / 65(80)$ \\
$1-3$ (confined to bone) & $46 / 51(90.2)$ & $20 / 24(83.3)$ & $17 / 19(89.5)$ \\
$4-5$ (extension to soft tissue) & $44 / 58(75.9)$ & $17 / 19(89.5)$ & $27 / 39(69.2)^{*}$
\end{tabular}


TMP-SMX treatment: one was classified a failure due to permanent disability, deformation of the toe, and the other patient required 32 weeks of treatment (TMP-SMX was an antibiotic used throughout the entire treatment course). No compliance concerns were noted. It is probable that this patient was actually a chronic osteomyelitis patient successfully treated with TMP-SMX and exemplifies the imperfect system of classifying acute and chronic infections; however, for the purposes of this study, this patient is considered a treatment failure for acute osteomyelitis.

\section{Discussion}

We acknowledge that our cure rate is much lower than typical cure rates found in the literature. This is because our definition, clearance of the infection within 14 weeks of treatment onset without recurrence within 30 days or permanent disability, is much more restrictive than the standard definition of The absence of clinical evidence of osteomyelitis after the follow-up period. ${ }^{9}$ The new definition emphasizes timely and complete return to normal function, which are very important factors for pediatric patients and their families, rather than cure from simply a microbial perspective.

We also report osteomyelitis of the skull associated with a significantly increased failure rate $(\mathrm{P}=0.0012)$. This association has not been reported in the current literature and should be a topic of further investigation, ideally with a larger sample size.

The presence of a prior bone defect, which was classified via denotation in radiological imaging reports, is highly correlated with treatment failure $(\mathrm{P}<0.00001)$; however, because permanent disability is a sign of treatment failure in our study, the likelihood of a complete recovery is almost not expected in this population due to prior damage of the site compounded by infection.

The most common organisms causing osteomyelitis in the literature are $S$. aureus, Streptococcus and gram negative organisms. The prevalence of $S$. aureus in this study, $58.6 \%$, is lower than the $70-90 \%$ reported in the literature; however, streptococcus and gram negative organisms were the next most common pathogens in both the study and the literature. ${ }^{4}$ MRSA specifically is reported in 9-30\% of the osteomyelitis cases in the literature, consistent with our study's finding of $18.8 \%{ }^{4}$ While the literature asserts that MRSA and Streptococcus pneumoniae are pathogens associated with increased failure rates, there were no significant differences in cure rates between pathogens in this study. ${ }^{4}$ As described in Table 3, MRSA had a cure rate of $76 \%$, which is not significantly different from non-MRSA cure rate $(80.6 \%, \mathrm{P}=0.815)$. This contradictory finding to the literature merits additional investigation.

The extent of infection, logically, is inversely proportional to the treatment success rate. Once the infection has extended past the bone to include soft tissue (stage 4 or 5 infection in our study), there is a concordant decrease in success rate. Patients with both an ESR $\geq 70$ and an infection with soft tissue involvement ( $\geq$ hematogenous stage 4) have an increased failure rate compared to patients with an $\mathrm{ESR}<70$ and an infection without soft tissue involvement $(\mathrm{P}<0.02)$. These two factors (ESR $\geq 70$ and soft tissue involvement), particularly when combined, likely represent more severe disease and therefore are potential clinical indicators for more aggressive management of the infection. Neither the ESR $\geq 70$ nor the soft tissue involvement are indicated as potential signs for treatment failure in the current literature; therefore, we recommend further studies to examine the relationships between ESR vs. cure rate and soft tissue involvement $v s$. cure rate, both separately and combined.

\section{Length of treatment and cure}

There is statistically significant increase in length of treatment time for patients who had at least one I\&D; they had, on average, at least 3 weeks longer antibiotic therapy. Unexpectedly, there is no significant difference in cure rate; this speaks to the effectiveness of the procedure because the patients who received an $I \& D$ generally had a more severe infection.

The average length of treatment, $10 \pm 0.76$ weeks overall (an average of $13.21 \pm 2.64$ weeks of treatment for contiguous infections and an average of $9.23 \pm 0.68$ weeks for hematogenous) is considerably longer than the guideline length of 4-6 weeks. This is likely due to a number of factors including complex co-morbidities, history of frequent infections, treatment compliance concerns, and patients presenting with an infection on the border between acute and subacute. The University of Michigan in particular receives a preponderance of referrals for such complicated osteomyelitis cases, likely contributing to this extended treatment duration.

\section{Bioavailability and cure}

Our study indicates transition from high bioavailability to low bioavailability antibiotics occurring $\geq 6$ weeks of treatment is associated with an increased likelihood of failure. This is not surprising because acute osteomyelitis is generally treated for 6 weeks, so any patient receiving greater than 6 weeks of treatment to begin with has likely already displayed increased clinical suspicion for treatment failure. As for determining if there is a minimum length of time that an osteomyelitis patient needs to stay on high bioavailability antibiotics, our findings show that early transition to low bioavailability antibiotics is not associated with an increased treatment failure rate.

\section{Therapy with trimethoprim-sul- famethoxazole}

While TMP-SMX is known to be an effective agent for MRSA treatment of skin and soft tissue infections, its efficacy for MRSA osteomyelitis has not been well documented. ${ }^{1-}$ ${ }^{3,5,6}$ Here we reviewed 17 patients treated for $\geq 7$ days with TMP-SMX, documenting a success rate of $88.2 \%$. This success rate is comparable to the success rate of the traditional clindamycin therapy, $75 \%(\mathrm{P}=0.423)$. Therefore, we recommend further investigation to confirm the efficacy of the use of TMP-SMX as an effective antibiotic option for treatment of MRSA acute osteomyelitis.

\section{Limitations}

The limitations of the study include that it is a retrospective study. Therefore, the analysis of the ideal time to transition from high to low bioavailability did not include large groups of equal number transitioning at preset timeframes. Due to the retrospective nature of the study, we were unable to determine the specific reason each patient was, or was not, transitioned from high to low bioavailability antibiotics; however, physician preference is suspected to be a major factor. Additionally, the number of patients with skull osteomyelitis was small $(n=6)$; therefore, the high failure rate of these patients should be the subject of further investigation.

\section{Conclusions}

The objectives of this study were to evaluate the use of TMP-SMX as a treatment option for pediatric acute osteomyelitis, to determine when and if there is an ideal point in treatment to switch from high bioavailability antibiotics to low bioavailability, and to determine if there are any other clinical risk factors that are indicative of potential treatment failure (therefore require more aggressive treatment).

In terms of TMP-SMX, it appears to be an acceptable antibiotic option to treat acute osteomyelitis and further studies evaluating its use are warranted. Such investigation is of the utmost importance due to the increasing prevalence of resistant $S$. Aureus such as MRSA and clindamycin resistant MRSA.

In terms of the ideal time point one should switch from high to low bioavailability antibiotics, there does not appear to be a minimum 
length of high bioavailability antibiotic treatment required for successful recovery. However, the preponderance of confounding variables leaves us unable to recommend an optimal point to switch from high to low bioavailability antibiotics at this time.

We were able to determine that a prior bone defect noted on imaging, more advanced disease noted on imaging, initial $E S R \geq 70$, the need for debridement, or skull osteomyelitis are all factors associated with increased failure rate. Therefore, these findings may be indications for more aggressive management of the infection and are important areas of future study.

\section{References}

1. Lew DP, Waldvogel FA. Osteomyelitis. Lancet. 2004;364:369-79.

2. Peltola H, Pääkkönen M. Acute osteomyelitis in children. $\mathrm{N}$ Engl $\mathrm{J}$ Med
2014;370:352-60.

3. Sia IG, Berbari EF. Osteomyelitis. Best Pract Res Clin Rheumatol 2006;20:106581.

4. Yeo A, Ramachandran M. Acute haematogenous osteomyelitis in children. BMJ 2014;348:66-74.

5. Chihara S, Segreti J. Osteomyelitis. Dis Mon 2010;56:6-31.

6. Conrad DA. Acute hematogenous osteomyelitis. Pediatr Rev 2010;31:464-71.

7. Stephen RF, Benson MKD, Nade S. Misconceptions about childhood acute osteomyelitis. J Child Orthop 2012;6:353-6.

8. Weichert S, Sharland M, Clarke NM, Faust SN. Acute haematogenous osteomyelitis in children: is there any evidence for how long we should treat? Curr Opin Infect Dis 2008;21:258-62.

9. Lazzarini L, Lipsky BA, Mader JT. Antibiotic treatment of osteomyelitis: what have we learned from 30 years of clinical trials. Int J Infect Dis 2005:9:127-38.

10. Liu RW, Abaza H, Mehta P, et al.
Intravenous versus oral outpatient antibiotic therapy for pediatric acute osteomyelitis. Iowa Orthop J 2013;33:20812.

11. Stanitski CL. Changes in pediatric acute hematogenous osteomyelitis management. J Pediatr Orthop 2004;24:444-5.

12. Cyriac JM, James E. Switch over from intravenous to oral therapy: a concise overview. J Pharmacol Pharmacother 2014;5:83-7.

13. Zaoutis T, Localio AR, Leckerman K, et al. Prolonged intravenous therapy versus early transition to oral antimicrobial therapy for acute osteomyelitis in children. Pediatr 2009;123:636-42.

14. Messina AF, Namtu K, Guild M, et al. Trimethoprim-sulfamethoxazole therapy for children with acute osteomyelitis. Pediatr Infect Dis J 2011;30:1019-21.

15. Pezone I, Leone S. Role of trimethoprimsulfamethoxazole for treatment of acute osteomyelitis in children. Pediatr Infect Dis J 2012;31:660-1. 